\title{
ON THE ALIGNMENT OF T TAURI STARS WITH THE LOCAL MAGNETIC FIELD
}

\author{
Gaspard Duchêne \\ Department of Physics 83 Astronomy, UCLA, Los Angeles, CA 90095-1562, USA \\ duchene@astro.ucla.edu \\ Francois Ménard \\ Laboratoire d'Astrophysique, Observatoire de Grenoble, F-38041 Grenoble cedex 9 \\ menard@obs.ujf-grenoble.fr
}

\begin{abstract}
Magnetic field is believed to play an important role in the collapse of a molecular cloud. In particular, due to the properties of magnetic forces, collapse should be easier along magnetic field lines, as supported by the large-scale sheet-like structure of the Taurus giant molecular cloud for instance. Here we investigate whether such a prefered orientation for collapse is present at a much smaller scale, that of individual objects. We use recent high-angular resolution images of $\mathrm{T}$ Tauri stars located in the Taurus star-forming region to find the orientation of the symmetry axis of each star+jet+disk system and compare it to that of the local magnetic field. We find that i) the orientations of the symmetry axis of $\mathrm{T}$ Tauri stars are not random with respect to the magnetic field, and ii) that young stars that are associated to a jet or an outflow are oriented very differently from those which do not have a detected outflow. We present some implications of this puzzling new result.
\end{abstract}

\section{Introduction}

Stars form in molecular clouds as a consequence of the gravitational collapse of dense molecular cores. This process is however sensitive to other phenomena than gravity, such as rotation or magnetic field. For instance, neutral species are only affected by gravity while ions are tightly bound to the magnetic field. Friction between ions and neutrals, known as ambipolar diffusion, modifies the kinematics of the neutrals, leading to protostars that are surrounded by pseudo-disks, even in the absence of rotation (Galli \& Shu, 1993). In the presence of straight magnetic field lines threading the cloud, Galli \& Shu (1993) showed that the ma- 
jor axis of the pseudo-disks are perpendicular to the direction of the original magnetic field, as expected if the collapse occurs preferentially along the field lines. The orientation of young stellar objects (YSOs) can therefore inform us directly on the importance of magnetic field in the collapse process. In the event of a leading role for magnetic forces, we expect circumstellar disks to be oriented so that their symmetry/rotation axis is (roughly) parallel with the local magnetic field in the cloud. On the other hand, if magnetic field does not influence much the collapse, disks might well be oriented at random.

In the Taurus-Auriga giant molecular cloud, both the dense gas clouds and the YSOs shows a sheet-like structure fully consistent with a large scale collapse along the magnetic field lines (Goodman et al. 1990; Hartmann 2002). At the individual object scale, Lee \& Myers (1999) showed that pre-stellar cores were elongated, with an average aspect ratio of $\sim 2.4$. If one attributes this elongation to rotation, it can be shown that their major axis are also preferentially found perpendicular to the magnetic field (Hartmann 2002). These findings support the case for a causal link between the direction of the large scale magnetic field and the orientation of young stars.

With the advent of high-angular resolution imaging devices, it is now possible to trace the orientation of already-formed YZOs. First, collimated jets and outflows from Myr-old T Tauri stars are commonly observed and can be used to trace their orientation. Strom et al. (1986) noted, during a deep imaging survey of Taurus sources driving HerbigHaro objects, that jets and outflows have a tendency to align parallel with the local magnetic field. MHD models predict that the ionised atomic jets of young stars are expelled perpendicular to the accretion disk (e.g., Shu et al. 1995; Ferreira \& Pelletier 1995), in agreement with striking high resolution observations of objects like HH 30 (Burrows et al. 1996). The preferred jet orientation therefore suggests that the rotation axis of T Tauri circumstellar disks are parallel to the local magnetic field. A similar conclusion was reached by Tamura \& Sato (1989) who found linear polarisation vectors of YSOs to be preferentially parallel to the local magnetic field. Although the interpretation of this result is affected by a $90^{\circ}$ ambiguity, it has reinforced the general belief that the orientation of young stars is in some way related to the direction of the local magnetic field.

Since early surveys for optical jets from T Tauri stars, many new jets and outflows have been identified. Furthermore, it is now also possible to spatially resolve circumstellar disks and accurately estimate their orientation through high angular resolution imaging. It is therefore time to readress this issue and study the link between the orientation of the 
local magnetic field and the orientation of YSOs in more detail. We will focus here on the well-studied Taurus star-forming regions.

\section{Direction of the magnetic field in Taurus}

In the presence of a magnetic field, elongated interstellar grains tend to spin along a preferred direction, with their axis of smallest moment of inertia parallel to the magnetic field (e.g., Lazarian 2002, and references therein). Dichroism is induced as the grains act like a picket-fence to absorb the light of background stars more efficiently along the direction perpendicular to the grains' rotation axis, i.e., generally perpendicular to the field. A net linear polarisation results in the other direction, i.e., parallel to the projected direction of the magnetic field.

This technique has been used by numerous authors to infer the structure of the magnetic field in molecular clouds. For our analysis, we have compiled over 400 linear polarisation measurements of background stars in Taurus (left panel in Fig. 1) from Vrba et al. (1976), Heyer et al. (1987), Moneti et al. (1987), Tamura et al. (1987), Tamura \& Sato (1989) and Goodman et al. (1990, 1992). To estimate the direction of the magnetic field at the location of each star in our sample (see below), we searched all the interstellar polarisation data in increasingly larger circles of radii $0.5-2.0^{\circ}$. We selected the smallest zone containing at least 4 different measurements of the magnetic field and retained the median of the measured position angles as the direction of the local magnetic field. As can be seen in Fig. 1, the polarisation vectors form a smooth structure throughout the region and we estimate that the orientation of the local magnetic field can be estimated to within $5^{\circ}$ for most objects.

\section{Orientation of $\mathbf{T}$ Tauri stars in Taurus}

We have first compiled a complete list of T Tauri stars in the TaurusAuriga star forming region from the Herbig \& Bell (1988, hereafter HBC) catalogue. We restrict our study to the zone $4 \mathrm{~h} 00<\alpha<5 \mathrm{~h} 00$ in right ascension and $+17^{\circ}<\delta<+30^{\circ}$ in declination. This yield a sample of over 100 pre-main sequence objects, to which we added HH 30 and IRAS 04158+2805 (Ménard et al. 2003), which are considered as normal $\mathrm{T}$ Tauri stars except for their edge-on circumstellar disk. They are not present in the HBC catalogue because of their extreme faintness induced by the occulting presence of their opaque disks. There might be a limited number of additional objects that we did not include in our study but we believe that they would not statistically affect our conclusions.

For each objects, we searched the litterature for the presence of a spatially resolved jet and/or disk. Only morphological evidences were used 


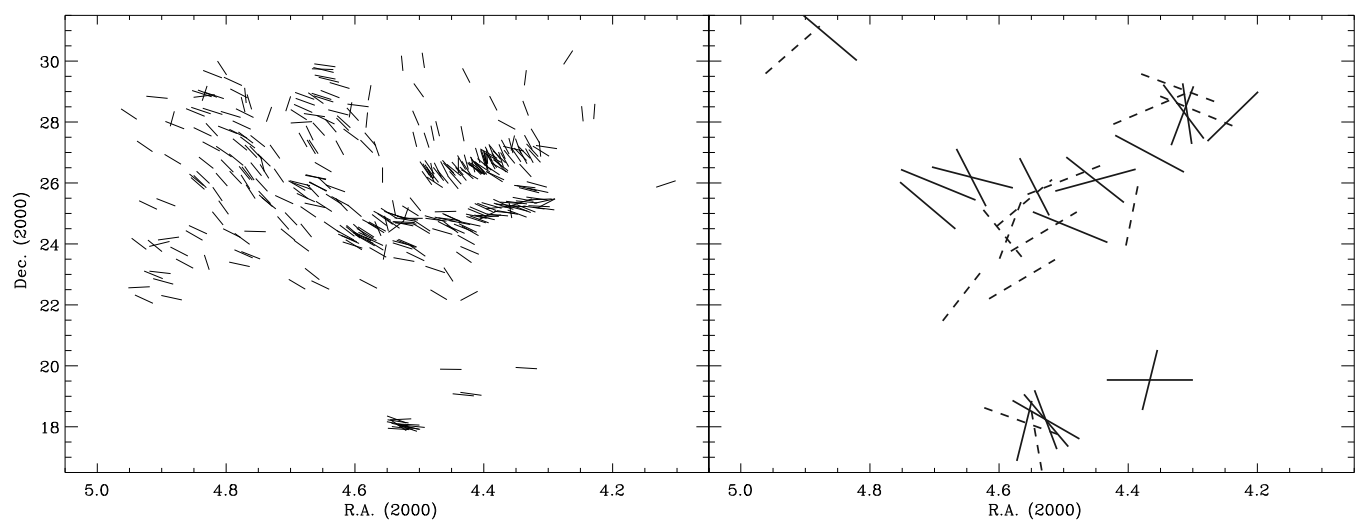

Figure 1. Left: Polarization measurements for background stars seen through the Taurus molecular cloud, tracing the direction of the magnetic field in the cloud. Only well defined measurements $\left(P / \sigma_{P}>3\right)$ are considered here. The length of all vectors is uniform and not proportional to the polarisation rate. Right: Orientation of $\mathrm{T}$ Tauri stars in Taurus as defined by an optical jet or outflow (solid vectors) or by a spatially resolved circumstellar or circumbinary disk (dashed vectors). In both cases, the symmetry axis of the system is shown.

in this process. Collimated jets are usually identified in deep narrowband images obtained at the wavelength of optical forbidden lines (e.g., Mundt et al. 1991; Dougados et al. 2000) or through long slit spectroscopy observations, which locate the redshifted and blueshifted parts of the jet on opposite sides of the star (e.g., Hirth et al. 1997). In most cases, jets are clearly resolved and their position angle is known within $10^{\circ}$ or better. Disks around young stars can be identified in two main ways: thermal imaging in the submillimeter and millimeter ranges and scattered light imaging in the optical and near-infrared. Because current instruments have a limited dynamic range, the latter technique is usually more sensitive to edge-on disks (e.g., Burrows et al. 1996) and to circumbinary rings (e.g., Roddier et al. 1996). In the millimeter continuum, disks appear as sources of thermal emission that are spatially resolved when observed with long baseline interferometers (e.g., Dutrey et al. 1996; Kitamura et al. 2002). Furthermore, observations of some disks in CO lines at millimeter wavelength reveal velocity profiles that are consistent with Keplerian rotation (e.g., Simon et al. 2000). When available, we used these resolved $\mathrm{CO}$ maps to define the orientation of the disk's semi-major axis. Disk orientations are known to within $5^{\circ}$ or better when a Keplerian velocity gradient is detected and within $5-15^{\circ}$ otherwise. More details on the individual sources as well as data tables are presented elsewhere (Ménard \& Duchêne 2003). 


\section{Relative orientations of T Tauri stars}

Among our targets, we identified 34 objects with a resolved jet and/or disk, including 12 that possess both of them. Among those 12 sources, all but one dubious case (DO Tau, see Ménard \& Duchêne 2003) have perpendicular jets and disks to within $20^{\circ}$ or better, as illustrated by the prototypical object HH 30 (Burrows et al. 1996). The symmetry axis of the system is determined by the orientation of the jet if it is present. Otherwise, we assume that the symetry axis lies in a direction perpendicular to the semi-major axis of the disk. As for the magnetic field direction, we only find the projection in the plane of the sky of the symmetry axis of the object. Despite possible projection effects, which we discuss below, it is worthwhile searching for possible correlations, which would trace an underlying physical link between the orientation of YSOs and the magnetid field.

The orientation of the symmetry axis for the $34 \mathrm{~T}$ Tauri stars for which we could determine it is plotted in the right panel of Fig 1 with different symbols for objects with and without jets. We plot the same axis, i.e. the symmetry axis of the star+jet+disk system, for both categories of objects. Although the direction of the symmetry axes shows significant scatter, two general trends can be identified from Fig 1, especially in the central area of the figure (R.A. $\sim 4$ h30, Dec. $\sim 25^{\circ}$ ). First, T Tauri stars for which a jet or outflow has been spatially resolved (solid vectors) are majoritarily oriented along the same direction as the local magnetic field. Second, T Tauri stars without a jet or an outflow but with a spatially resolved circumstellar disk are roughly oriented perpendicularly to the local magnetic field. While the former conclusion is similar to those reached in past studies, the latter point is revealed here for the first time. We now quantify it in more detail before exploring its implication for star formation studies.

For each object with a known orientation in the plane of the sky, we measured the "misalignment angle" between the axis of symmetry of the T Tauri star and that of the local magnetic field. A value of $0^{\circ}$ indicate that both are parallel while it is $90^{\circ}$ if they are perpendicular. The distributions of these angles for both our subsamples (objects with and without a spatially resolved jet) are shown in Fig. 2 (left panel). The two histograms are statistically different at the $\sim 3 \sigma$ level: there is a significant difference in the respective orientations of TTauri stars with and without jets. Systems with a jet have their symmetry axis roughly parallel to the local magnetic field (median angle $=25^{\circ}$ ) while

systems without a jet or an outflow are preferentially perpendicular to it (median angle $=75^{\circ}$ ). Interestingly, the distribution of orientations for 

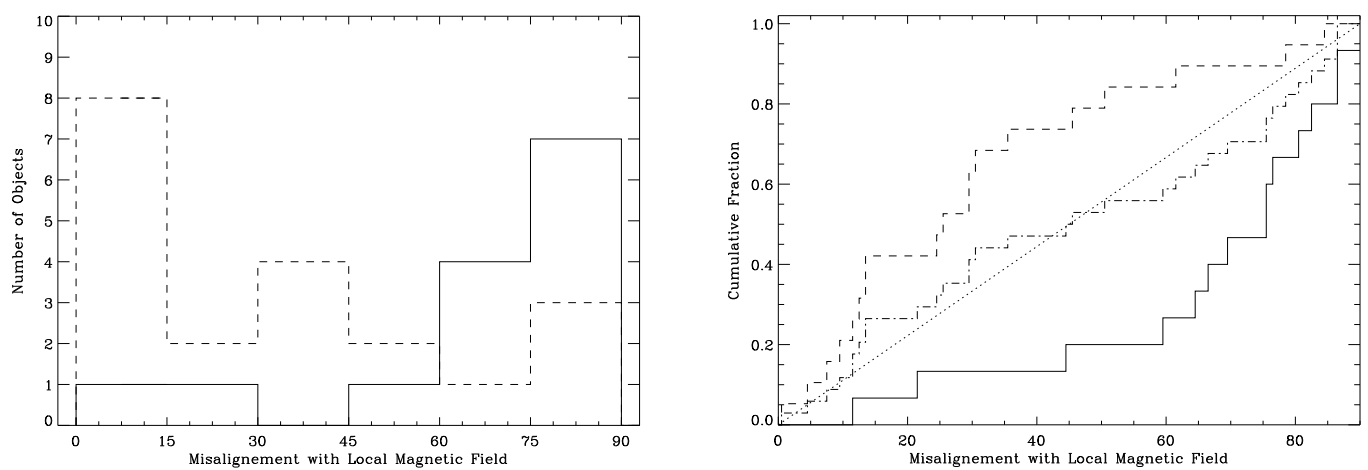

Figure 2. Left: Distribution of the angle between the orientation of the symmetry axis of T Tauri stars and their local magnetic field. From $0^{\circ}$ to $90^{\circ}$, these two directions go from parallel to perpedicular. The dashed histogram represent T Tauri stars with a jet or an outflow while the solid histogram are those for which only a disk could be spatially resolved. Right: Cumulative distributions of angles between the symmetry axis of T Tauri stars and their local magnetic field for objects with a jet/outflow (dashed), with only a disk (solid) and for the whole sample of objects with known orientation (dot-dashed). The dotted line represents the expected distribution if YSOs were randomly oriented with respect to the local magnetic field.

our complete sample of 34 objects is consistent with random orientation, as illustrated in the right panel of Fig. 2.

As pointed out earlier, our sample is unlikely to be $100 \%$ complete and an unknown selection effect may be responsible for this apparent difference. However, the imaging studies used to build our database of stellar orientations are equally sensitive to structures (jets, outflows, disks) in any direction in the plane of the sky. It is difficult to think of a selection effect that would preferentially sample stars oriented in a specific direction. Although there are strong selection effects regarding inclination, in particular against pole-on systems, we do not find any significant difference between the distributions of inclinations of objects with and without a jet. Therefore, we do not believe that the distinction between the two subsamples compared here can be distinguish by specific geometric configurations that lead to undetectable jets for some YSOs.

\section{Open questions}

The fact that $\mathrm{T}$ Tauri stars do not orient at random with respect to the magnetic field and that the presence or absence of a bipolar jet is statistically linked to the orientation of the object clearly shows that the magnetic field does play an important role in the collapse of individual dense cores and their subsequent evolution. The origin of the differential 
orientation trend discovered here is not clear yet and only suggestions can be put forward at this point. A first important point to note is that, while jets are most likely launched through a specific magnetic configuration linking the star, its accretion disk and the jet, this involves the stellar magnetic field. On the other hand, our studies of orientations concerns the molecular cloud magnetic field, which may have a different topology and orientation.

First, it is unclear whether T Tauri stars retain the same orientation throughout their evolution. It could be that the collapse is strongly driven by the magnetic field, with all systems having their symmetry axis roughly parallel to the local magnetic field, and that some of them later become misaligned through an unknown process. It is worth noting that the orientation of bipolar outflows from more embedded (i.e., younger) Class I sources in Taurus show the same tendency to align parallel to the magnetic field as T Tauri stars that possess a jet (see Ménard \& Duchêne 2003). If all YSOs retain their original orientation, where are the precursors of those T Tauri stars that now only have a disk? Despite this suggestive trend for protostars, we fail to identify any mechanism that could provide enough torque to rotate by $90^{\circ}$ a star+disk system.

A second possibility is that YSOs are formed with random orientations, as suggested by the distribution of our complete sample, and that subsequent evolution lead to the formation of a powerful jet/outflow only in some conditions. For instance, Ferreira (1997) showed that a quadrupolar magnetic field configuration in the disk lead to a much weaker disk-wind, if any, than a dipolar configuration could achieve. We can then propose that the configuration of the stellar magnetic field will be dipolar if its axis of symmetry is more or less aligned with the local field in the molecular cloud, while only quadrupolar (or high order configurations) can be created if the relative orientation is close to perpendicular. This could be the consequence of a feedback of the cloud magnetic field on the (currently unknown) growth mechanism of the T Tauri star field. The fact that Class I sources do not apper to be randomly oriented is somewhat problematic in this picture though.

A third possible explanation to the observed trend is a projection effect, in which $\mathrm{T}$ Tauri stars that have a jet are located in a different part of the cloud than those who do not have one. The latter could be seen in projection over the Taurus cloud, while being located well in front, where the magnetic field orientation could be different. In that case however, the field at the periphery of the cloud would need to be twisted by $90^{\circ}$ with respect to the field found inside the cloud where T Tauri stars with jets would presumably be located. It is unclear why this would be the case. Furthermore, one would have to explain why the 
objects located in the outer parts of the cloud are systematically devoid of jets and outflows.

Only tentative interpretation of the observational result presented here can be given for now; none of them is fully satisfying. Conducting similar studies in other star-forming regions, such as $\rho$ Ophiuchus or Orion, and measuring submillimeter polarisation vectors from much younger prestellar cores will help understanding whether this trend is a general one and possibly identify other factors that play a role in this preferred alignment of objects with the magnetic field.

\section{Acknowledgments}

The authors wish to thanks the organizers of the conference for the quality of its program and its flawless organisation, as well as Philippe André, Bo Reipurth and Hans Zinnecker for stimulating discussions.

\section{References}

Burrows, C. J. et al. 1996, ApJ, 473, 437

Dougados, C. et al. 2000, A\&A, 356, L41

Dutrey, A. et al. 1996, A\&A, 309, 493

Ferreira, J. \& Pelletier, G. 1995, A\&A, 295, 807

Ferreira, J. 1997, A\&A, 319, 340

Galli, D. \& Shu, F. H. 1993, ApJ, 417, 243.

Goodman, A. A. et al. 1990, ApJ, 359, 363

Goodman, A. A. et al. 1992, ApJ, 399, 108

Hartmann, L. 2002, ApJ, 578, 914

Herbig, G. H. \& Bell, K. R. 1988, Lick Obs. Bulletin, No. 1111.

Heyer, M. et al. 1987, ApJ, 321, 855

Hirth, G. A., Mundt, R. \& Solf, J. 1997, A\&AS, 126, 437

Kitamura, Y. et al. 2002, ApJ, 581, 357

Lazarian, A. 2002, Elsevier preprint. (astro-ph/0208487).

Lee, C. W. \& Myers, P. C. 1999, ApJS, 123, 233

Ménard, F. et al. 2003, ApJ, in press.

Ménard, F. \& Duchêne, G. 2003, A\&A, submitted.

Moneti, A. et al. 1984, ApJ, 282, 508

Mundt, R., Ray, T. P. \& Raga, A. C. 1991, A\&A, 252, 740

Roddier, C. et al. 1996, ApJ, 463, 326

Shu, F. H. et al. 1995, ApJ, 455, L155

Simon, M., Dutrey, A. \& Guilloteau, S. 2000, ApJ, 545, 1034

Strom, K.M. et al. 1986, ApJS, 62, 39

Tamura, M. et al. M. 1987, MNRAS, 224, 413

Tamura, M. \& Sato, S. 1989, AJ, 98, 1368

Vrba, F. J., Strom, S. E. \& Strom, K. M. 1976, AJ, 81, 958 